\title{
Research on Preparation and Photocatalytic Activity of Fe-doped $\mathrm{TiO}_{2}$
}

\author{
Nanotube \\ Shuai Zhang ${ }^{1, a}$, Yongtao Liü ${ }^{1, b}$, Binbin Su ${ }^{1, c}$ \\ ${ }^{1}$ Department of Micro and Nanosystems Technology, University College of Southeast \\ Norway, borre 3184, Norway \\ ${ }^{\mathrm{a}}$ email, ${ }^{\mathrm{b}}$ email, ${ }^{\mathrm{c}}$ email
}

Keywords: $\mathrm{TiO}_{2}$ nanotube, Fe- doping, Photocatalytic Activity

\begin{abstract}
Taking iron nitrate as iron source, Fe-doped $\mathrm{TiO}_{2}$ nanotube arrays with $\mathrm{Fe}^{3+}$ inclusion were synthesized by using anodic oxidation treatment. Simultaneously, the photocatalytic activity of this catalyst was measured by using photo-induced degradation of methyl orange. The results showed that $\mathrm{Fe}^{3+}$ inclusion could effectively enhance the photocatalytic activity of $\mathrm{TiO}_{2}$ nanotube.
\end{abstract}

\section{Introduction}

As a result of the semiconductor multi-phase photocatalytic reactor, it has become an ideal environment pollution control and clean energy production technology because of its characteristics, which can be used for the reaction at room temperature and the direct use of solar energy. With the increasing of environmental pollution and energy crisis, the effective application of this technology is one of the main research focuses in the field of modern science and technology. For semiconductor photocatalysis are mostly metal oxides and sulfides, which $\mathrm{TiO}_{2}$ semiconductor has a non-toxic, non-corrosive, and can be used repeatedly, can be completely mineralized to organic pollutants in water, the advantages of $\mathrm{CO}_{2}$ and inorganic ions, no two pollution, has incomparable traditional high temperature, the conventional catalytic technology and adsorption technology attractive charm, attracted widespread attention in the technical aspects of green environmental governance. $\mathrm{TiO}_{2}$ was used as photocatalyst in the form of powder, thin film, and carrier composite materials. In the initial stage of the development of photocatalytic, the research mostly focused on the $\mathrm{TiO}_{2}$ powder. This is because the $\mathrm{TiO}_{2}$ powder preparation technology is more mature, and the photocatalytic suspension system is simple and convenient, can make the $\mathrm{TiO}_{2}$ powder and the degradation of the more adequate contact. But the powder of $\mathrm{TiO}_{2}$ photocatalyst in the use process is not easy to disperse, easy to agglomerate and difficult to recycle, the light scattering is strong, there is even the possibility of contamination two times, and resulting in promotion difficult large-scale application and commercialization by $\mathrm{TiO}_{2}$ photocatalytic technology based powder. The preparation of $\mathrm{TiO}_{2}$ tubular materials, not only increases as the surface area of the catalyst, contribute to the adsorption degradation, and unique structural properties of $\mathrm{TiO}_{2}$ tubes and good crystal structure, the transfer of the interface between the electronic charge vector provides a good soak path. Therefore, the application of $\mathrm{TiO}_{2}$ tubes in the photocatalytic technology has attracted much attention of many researchers. In this experiment, industrial pure titanium substrates, ammonium fluoride, glycerin mixed electrolyte. $\mathrm{Fe}\left(\mathrm{NO}_{3}\right)_{3}$ as the source of iron, iron doped tube arrays prepared by anodic oxidation method, and methyl orange as object, the effects of doped $\mathrm{TiO}_{2}$. 


\section{Experiment}

Preparation of Fe-doped $\mathbf{T i O}_{2}$ Nanotube. The industrial pure titanium $(25 \mathrm{mmX} 15 \mathrm{~mm})$ were polished with emery paper, successively with acetone, ethanol and distilled water for ultrasonic cleaning and air, and then immersed in the volume ratio of $\mathrm{V}(\mathrm{HF})$ : $\mathrm{V}\left(\mathrm{HNO}_{3}\right)$ : $\mathrm{V}\left(\mathrm{H}_{2} \mathrm{O}\right)=1: 4: 5$ mixed acid etching. Second, using deionized water wash, processing good chin in the air dried. The tablets will be pretreated as the working electrode. The platinum sheet as the electrode, anode and cathode respectively from DC power supply, the spacing is $3 \mathrm{~cm}$. with the mass fraction of ammonium fluoride, $0.5 \%$ different amounts of nitrate, iron sulfate and glycerol mixed solution as electrolyte at room temperature, magnetic stirring of anodic oxidation. At the rate of $17 \mathrm{mV} / \mathrm{S}$ DC voltage from $\mathrm{O}$ to $3 \mathrm{OV}$ slows reaction at constant pressure, 10h. When the reaction is finished, remove the surface of the water and wash the surface with the de ionized water. The specimen is placed in the Sx-4-10 box type resistance furnace was claimed. To $5 \mathrm{C} / \mathrm{min}$ temperature to 450 degrees Celsius, heat insulation $2 \mathrm{~h}$, cool to room temperature to remove the use of.

We used the DX-1000 FANGYUAN type X - ray diffraction analysis of the catalyst phase, Cook -ray, tube voltage $35 \mathrm{kV}$, tube current $25 \mathrm{~mA}$. N2 adsorption desorption isotherms were determined by the BuilderSSA-4200 type pore ratio surface analyzer, and the sample was measured at $300 \mathrm{C}$, and the absolute pressure was $6.65 \times 102 \mathrm{~Pa}$ under $3 \mathrm{H}$. The morphology of catalyst surface was observed by JEOLJSM-5900LUJAPAN scanning electron microscope. Multi-functional surface analysis of electron spectrometer test samples the elemental composition, chemical state and content of British Kato's company XSAM800, Al, X $(1486.6 \mathrm{eV})$ target light gun power is $12 \mathrm{kV}$ * $15 \mathrm{~mA}$, the base vacuum analysis room $2 \times 10-7 \mathrm{~Pa}$, using FAT mode, using Cu2p3/2 spectrometer (932.67eV) Ag3d5/2, (368.30eV), Au4f7/2 (84.00eV) calibration standard sample data using C1s $(284.8 \mathrm{eV})$ correction of carbon pollution. Thermal gravimetric differential scanning calorimetric (DSC - TG) was carried out with a NETZSCHSTA449C thermal analyzer, and the heating rate of /min at $10 \mathrm{C}$ in air was heated up to $800 \mathrm{c}$. Determination of UV - visible (Vis - UV) diffuse reflectance spectra by Hitachi U-3010, measuring range $220 \sim 800 \mathrm{~nm}, \mathrm{BaSO} 4$ for reference.

Measurement of Photocatalytic Activity. MB photocatalytic reaction was carried out in a self-made $500 \mathrm{~mL}$ cylindrical reactor. The reactor is 2 concentric cylindrical quartz glass container, outer package with aluminum foil, the middle suspension with ultraviolet lamp (quanta Lighting Electrical Appliance Factory 8W UV black light lamp, the main wavelength of 365nm), the reaction solution is placed on the inside and outside the apartment, in the reaction process from the bottom row to pass into the air, oxygen and stirring. Preparation of concentration of MB solution 200mL $12 \mathrm{mg} / \mathrm{L}$, catalyst $0.2 \mathrm{~g}$, light mixing ventilation $1 \mathrm{H}$ reached adsorption equilibrium after opening the UV lamp, every 20min sample, centrifugal separation (4000r/min, 5min), the absorbance meter was measured at the wavelength of 666nm with 721 type spectrophotometry according to the calculation, the solution concentration of Lambert - Beer law. Photocatalytic degradation rate equals $\left(\mathrm{c}_{\mathrm{t}}-/ \mathrm{c}_{0}\right) \mathrm{c}_{0}$ $100 \%$, total decolorization rate $=\left(1-c_{t} / 12\right) \times 100 \%\left(c_{0}\right.$ for the initial concentration of methylene blue solution after desorption equilibrium, $c_{t}$ for concentration). First photocatalytic reaction samples for unfired samples after the photocatalytic reaction, centrifugal separation, drying at 100 DEG 5h, 400 DEG C $2 \mathrm{H}$ roasting in muffle furnace was raised, the photocatalytic experiment used repeatedly next time.

\section{Results and Discussion}

Characterization of Fe-doped $\mathrm{TiO}_{2}$ Nanotube. The SEM images of Fe-doped $\mathrm{TiO} 2$ nanotube arrays are shown in Figure 1. As can be seen from the figure they have formed a tubular structure 
with only doped different array surface tube structure characteristics change. Different degrees at lower concentrations (Figure 1a) array surface arranged disorderly, uneven length. When the dosage of $0.05 \mathrm{~mol} / \mathrm{L}$, nanotube arrays exhibits orderliness and the regularity of the height of the mouth clean and smooth, the average diameter of $100 \mathrm{~nm}$, wall thickness is about $15 \mathrm{~nm}$. Compared with the nanotubes, the nanotubes have a slight increase in the diameter of the nanotubes. When the amount is higher than this value (Figure 1c and d) will observe the emergence of a large number of nozzle surface covering. This layer may be formed by the rupture of the thin tube at the top of the surface under the stress of the surface. The surface covering the mouth of nanotube arrays severely hinder the possibilities by blocking it in full contact with foreign minister. Through the analysis of the relative content of Fe doped $\mathrm{TiO}_{2} 450$ nanotube arrays calcined after $2 \mathrm{~h}$ elements spectrometer. The catalyst calcined is mainly composed of $\mathrm{Ti}$ and $\mathrm{O}$ elements of the $\mathrm{O} / \mathrm{Ti}$ mass ratio of iron containing elements of 1.49 atom fractions is $1.42 \%$. While the EDS is only qualitative determination of element content, but on the whole still can be seen on the surface element content. Iron doped $\mathrm{TiO}_{2}$ nanotube arrays showed excellent visible light photocatalytic activity, and the photocatalytic activity increased first and then decreased with the increase of Fe content. For general metal ions doping has an optimal value, too much or too little is not conducive to the improvement of photocatalytic activity. When the doping concentration of $\mathrm{Fe}^{3+}$ is smaller than that of the semiconductor, there are not enough carrier trapping traps in the semiconductor, and the effect is not obvious. When the doping concentration is too large, the distance between the semiconductor capture will be reduced, so that the electron hole recombination rate of increase.

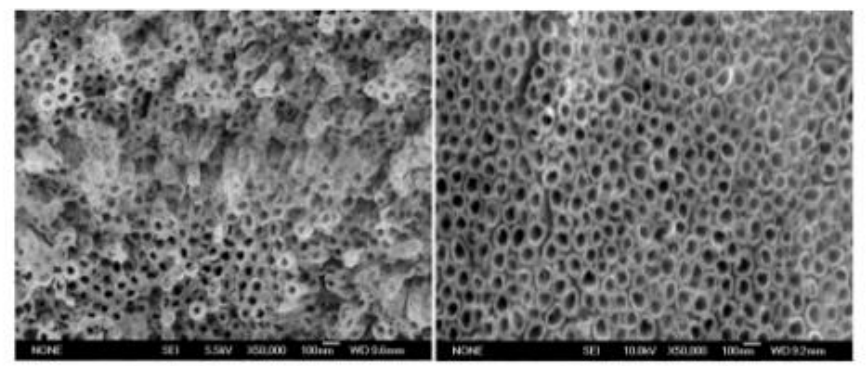

(a) $0.02 \mathrm{~mol} / \mathrm{L}$

(b) $0.05 \mathrm{~mol} / \mathrm{L}$

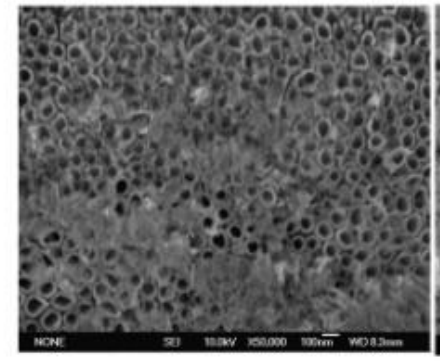

(c) $0.08 \mathrm{~mol} / \mathrm{L}$

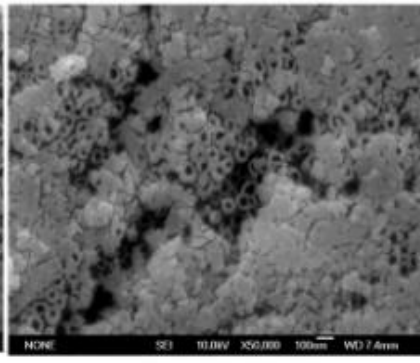

(d) $0.10 \mathrm{~mol} / \mathrm{L}$

Figure 1. SEM images of Fe-doped TiO2 nanotube arrays

Analysis of Photocatalytic Activity. The experimental results of photocatalytic oxidation of Fe doped TiO2 powders are shown in Figure 2 and Figure 3. The Figure 2 shows that the 1 catalyst in the photocatalytic reaction, with iron doping amount, the total decolorization of catalyst increased firstly and then decreased, but the total decolorization rate remained above 95\%, the maximum total amount of iron doped decolorization rate corresponding to $2 \%$. When the catalyst was 2 times the photocatalytic decolorization rate of the total samples used repeatedly, decreased, iron doping amount was $1 \%$, 3\% samples were decreased by $19.4 \%$ and $22.8 \%, 0,2 \%$ the amount of iron doped samples were decreased by $4.9 \%$ and $2.5 \%$, the best iron doping amount is $2 \%$. This can be 
explained as follows: The sample of the largest specific surface area, but its total pore volume minimum, small aperture, and the $\mathrm{MB}$ is relatively high molecular weight and small pore size disadvantage on adsorption of MB molecules, the total decolorization rate is low; while the sample mesoporous and macroporous, the total pore volume the largest, and the specific surface area of up to $117.618 \mathrm{~cm}^{3} / \mathrm{g}$, so the total decolorization rate maximum. In addition, when the doping concentration is small, there is not enough carriers trapping trap in the semiconductor, and the effect is not clear. However, when the concentration of doped ions increases to a certain degree, the doped ions will become the recombination center of the electron and hole. The total decolorization rate of $\mathrm{TiO}_{2}$ powder was decreased to $61.4 \%$, and the total degradation rate was $23.3 \%$ after 3 times. From the Figure 2, the iron content of the sample particle size of $2 \%$ is 0.1 to $0.2 \mathrm{mu} \mathrm{m}$, after the end of the photocatalytic experiment, most of the powder can be naturally settling in the $1 \mathrm{~h}$, and the recovery is convenient.

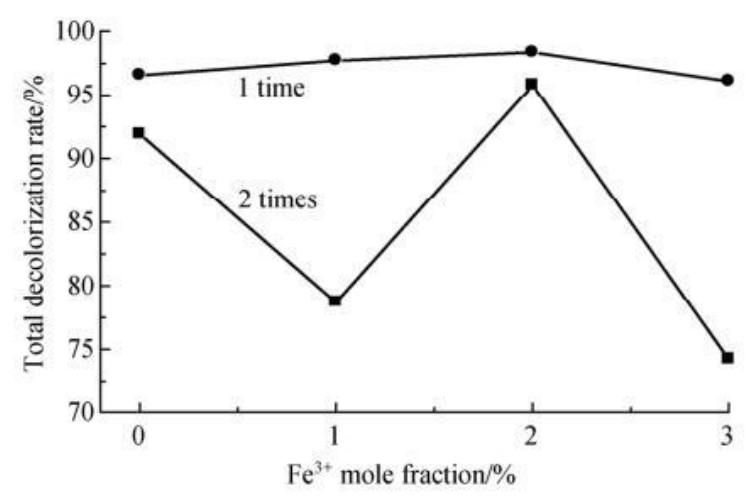

Figure 2. Effect of Fe content

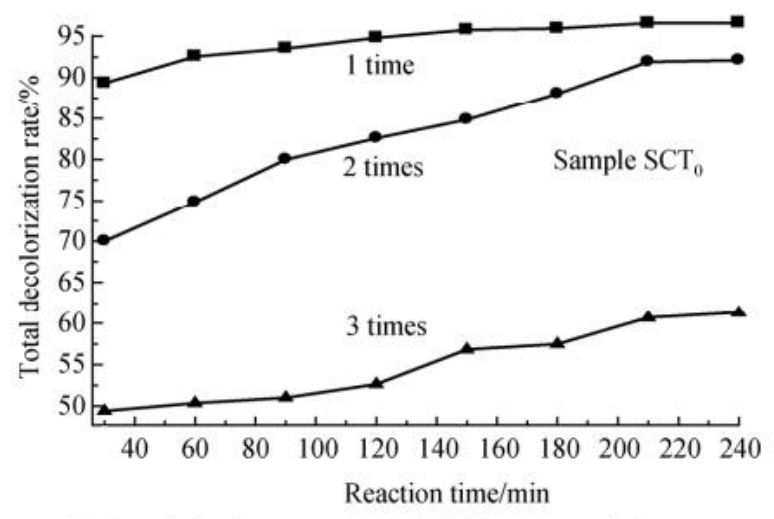

Figure 3. Decolorization rate of sample $\mathrm{SCT}_{0}$ vs. reused times

\section{Conclusion}

The application of photocatalysis in environmental management has attracted more and more attention. But there is still a certain distance from the experiment to the practical application. Most of the experiments need the powdered $\mathrm{TiO}_{2}$ and UV light source. Future research will try to solve the problems. With the deepening of the research of $\mathrm{TiO}_{2}$ photocatalysis, we believe to be able to develop efficient photocatalytic materials in the near future. 


\section{References}

[1] Tang Shouqiang, He Jingping, Zhang Zhao, Journal of the Chinese Ceramic Society, Vol. 40 (2012) No 7, p.950-956

[2] Fan Ding, Cui Wenjuan, Gu Yufen, Zhang Xinlei, Journal of Lanzhou University of Technology, Vol. 37 (2011) No 4, p.23-26

[3] Du Hongli, Zhang Zhaozhi, Li Yan, Hu Jiawen, Materials Review, Vol. 25 (2011) No 7, p.20-24

[4] Huang Yicao, Zhao Zhefei, Li Shixiong, Di Jing, Zheng Huajun, Chinese Journal of Inorganic Chemistry, Vol. 31 (2015) No 1, p.133-139 